Case Report

\title{
Retained Placenta Accreta Mimicking Choriocarcinoma
}

\author{
Maureen P. Kohi, ${ }^{1}$ Gabrielle A. Rizzuto, ${ }^{2}$ Nicholas Fidelman, ${ }^{1}$ \\ Jennifer Lucero, ${ }^{3}$ and Mari-Paule Thiet ${ }^{4}$ \\ ${ }^{1}$ Department of Radiology and Biomedical Imaging, University of California, San Francisco, San Francisco, CA 94143, USA \\ ${ }^{2}$ Department of Pathology and Laboratory Medicine, University of California, San Francisco, San Francisco, CA 94143, USA \\ ${ }^{3}$ Department of Anesthesia and Perioperative Care, University of California, San Francisco, San Francisco, CA 94143, USA \\ ${ }^{4}$ Department of Obstetrics, Gynecology and Reproductive Sciences, University of California, San Francisco, \\ San Francisco, CA 94143, USA \\ Correspondence should be addressed to Maureen P. Kohi; maureen.kohi@ucsf.edu
}

Received 15 July 2015; Accepted 10 September 2015

Academic Editor: George Adonakis

Copyright (C) 2015 Maureen P. Kohi et al. This is an open access article distributed under the Creative Commons Attribution License, which permits unrestricted use, distribution, and reproduction in any medium, provided the original work is properly cited.

This case demonstrates a rare event of retained invasive placenta masquerading as choriocarcinoma. The patient presented with heavy vaginal bleeding following vaginal delivery complicated by retained products of conception. Ultrasound and computed tomography demonstrated a vascular endometrial mass, invading the uterine wall and raising suspicion for choriocarcinoma. Hysterectomy revealed retained invasive placenta.

\section{Introduction}

Abnormally invasive placenta is a life-threatening condition that occurs when chorionic villi adhere to the uterine myometrium without normal intervening decidua basalis [1]. The most common complication of invasive placenta is postpartum hemorrhage $(\mathrm{PPH})$, which often requires hysterectomy [2].

Retained products of conception (RPOC) or placental fragments are a common cause of $\mathrm{PPH}$ with an incidence of $3 \%-5 \%$ after routine vaginal delivery [3]. Ultrasound (US) is the primary modality during the antepartum period and the modality of choice to evaluate for PPH [4]. Combined gray-scale and color Doppler US allow real-time assessment of the uterine cavity and blood flow, which aid in the diagnosis of RPOC. Early diagnosis is critical for directing clinical management and for preventing associated immediate complications, such as infection, as well as future obstetric complications [5].

Herein, we present a case of retained invasive placenta, which was undetected in the antepartum and mimicked choriocarcinoma in the postpartum period, ultimately resulting in hysterectomy.

\section{Case Report}

A 39-year-old gravida 3 nulliparous female with a dichorionic diamniotic twin pregnancy at 36 weeks and 4 days was admitted to our institution for induction of labor secondary to intrauterine growth restriction (IUGR) of both infants. Her obstetrical history was significant for two prior dilatation and curettage $(\mathrm{D} \& \mathrm{C})$ procedures. Her antenatal ultrasounds demonstrated two normal placentas without evidence of previa or placental invasion.

She progressed to spontaneous rupture of membranes 18 hours following administration of oxytocin and placement of a transcervical Foley balloon. Once US confirmed vertex lie of both infants, the patient was moved to the operating room (OR) where labor progressed normally with delivery of two female infants weighting $2085 \mathrm{~g}$ (Apgar scores of 8 and 8 at 1 and 5 minutes) and $1945 \mathrm{~g}$ (Apgar scores of 4 and 8 at 1 and 5 minutes), respectively.

The third stage of labor was complicated by retained placenta, which was extracted manually and with banjo curettage under ultrasound guidance. At the end of the procedure, a thin endometrium was confirmed by US. 


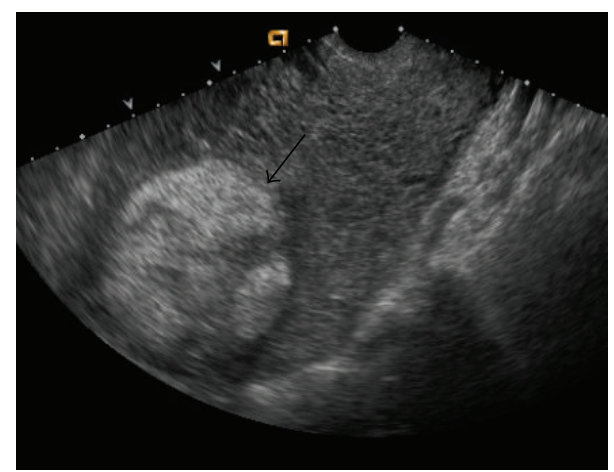

FIGURE 1: Gray-scale US demonstrates an echogenic mass in the endometrial cavity (black arrow).

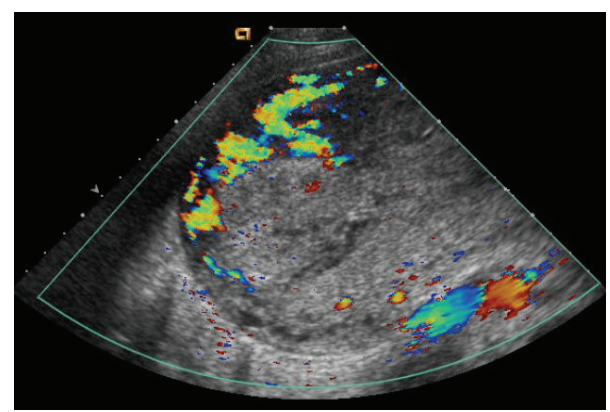

FIGURE 2: Color Doppler US image demonstrates vascularity in the echogenic mass with extensive vascularity surrounding the mass.

Initially, the postpartum course was uncomplicated, and the patient was discharged on postpartum day two in stable condition. However, on postpartum day five, while visiting her infants in the hospital, the patient passed an orange-sized blood clot. A repeat US demonstrated a thin endometrial stripe. For the next several weeks, light bleeding continued.

At the routine 6-week postpartum visit, the patient again passed a large blood clot and her uterus was palpable $2 \mathrm{~cm}$ below the umbilicus. At that time, serum quantitative human chorionic gonadotropin (hCG) level was $203 \mathrm{IU} / \mathrm{L}$, and hematocrit was $33 \%$.

Transvaginal US showed a large echogenic mass within the endometrial cavity, measuring $9.4 \times 8.5 \times 6.7 \mathrm{~cm}$ (Figure 1). Color Doppler US demonstrated vascularity, predominately in the periphery of the mass (Figure 2). Computed tomography (CT) demonstrated a large hypervascular and heterogeneously enhancing uterine mass measuring $10.4 \times 15 \times 16.8 \mathrm{~cm}$ with diffuse myometrial invasion (Figure 3 ) and CT chest demonstrated bilateral ground glass nodules (Figure 4). Differential diagnoses included gestational trophoblastic disease versus RPOC, but choriocarcinoma was favored given the hypervascularity noted on CT, the degree of uterine invasion, and the presence of pulmonary nodules, which is worrisome for metastases. RPOC was considered less likely in light of manual and instrumental placental extractions and thin stripe noted US performed in the OR.

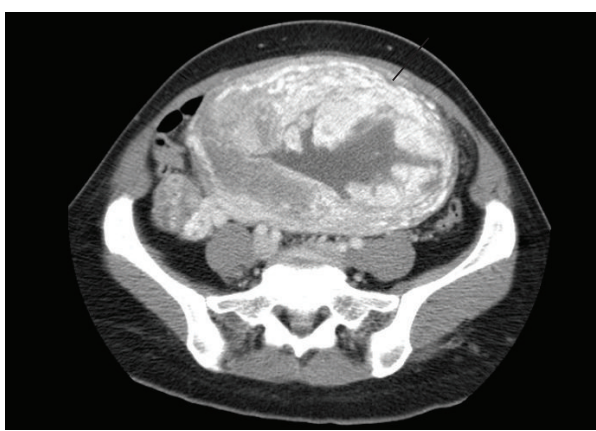

FIGURE 3: Contrast-enhanced CT image demonstrates hypervascular uterine mass. Note loss of plane between the mass and the uterine wall (white arrow).
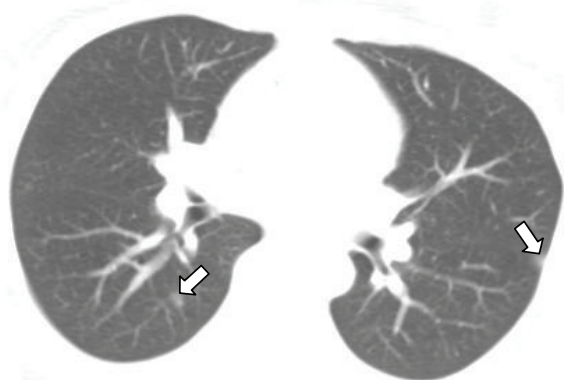

FIGURE 4: CT image of the chest demonstrates ground glass opacities in the lungs (open arrows).

Management options included transcervical biopsy for diagnosis (D\&C) with frozen section with subsequent hysterectomy in case of malignancy or outright hysterectomy. The patient and her husband did not desire future fertility and preferred hysterectomy.

The patient underwent total abdominal hysterectomy, and pathology demonstrated placenta accreta (Figures 5(a) and $5(\mathrm{~b})$ ). The postoperative course was uncomplicated, and the patient was discharged on postoperative day four.

\section{Discussion}

Invasive placenta is a condition caused by placental invasion into the uterine wall. Three distinct types of invasive placenta exist, based on the degree of placental villi invasion into the myometrium: placenta accreta (superficial invasion of the basalis layer), placenta increta (deeper invasion of the myometrium), and placenta percreta (deeper invasion involving the serosa and other surrounding organs such as the bladder) [6]. Risk factors for invasive placentation include placenta previa, previous history of cesarean delivery, advanced maternal age, previous uterine surgery, and multiparity [7]. The incidence of invasive placentation has increased fivefold over the past few decades from 1 in 2510 in the 1980s to currently about 1 in 533 pregnancies [7]. The major contributing factor to this is likely the increase in the rate of cesarean delivery and uterine instrumentation [8]. 


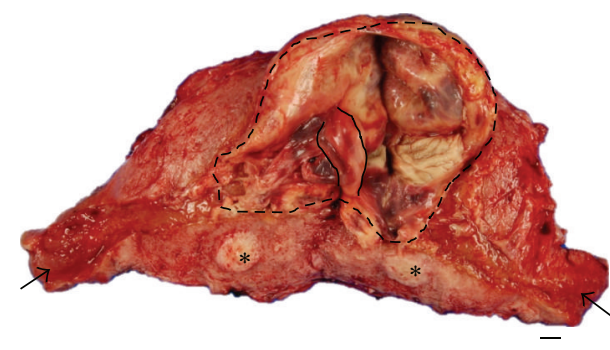

(a)

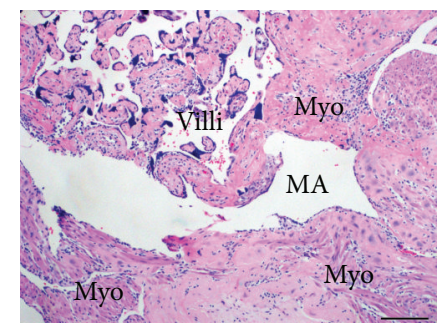

(b)

FIGURE 5: Grossly retained placenta with microscopic evidence of placenta accreta. (a) Gross photograph of hysterectomy specimen bisected in coronal plane shows $\sim 12 \times 11 \times 3 \mathrm{~cm}$ fundal placenta (outlined in dashed lines) with $\sim 3.5 \mathrm{~cm}$ umbilical cord (outlined in solid lines) (arrows at cervical os, $*=$ leiomyoma). (b) Hematoxylin and eosin stained microscopic section demonstrates degenerating placental villous parenchyma [villi] adjacent to large bands of myometrial smooth muscle [myo] without intervening decidua [MA = maternal myometrial artery]. Scale bars: (a) $1 \mathrm{~cm}$ and (b) 100 microns. Note: due to extensive tissue degeneration at the placenta/myometrial interface, the depth of accreta could not be accurately determined on pathologic examination.

Antenatal diagnosis of invasive placentation is critical and has been shown to decrease maternal morbidity [9]. US is the primary imaging modality for the diagnosis of invasive placenta in the antepartum period [10] with sensitivity of $91 \%$ and specificity of $97 \%$ [11]. Findings suggestive of invasive placentation on US include intraplacental lacunar spaces, lack of normal retroplacental clear zone, irregularity and attenuation of the uterine-bladder interface, retroplacental myometrial thickness, and bridging vessels between the placenta and bladder wall when using color Doppler [12].

RPOC refers to intrauterine tissue that persists after delivery or termination of pregnancy and is often of placental trophoblastic origin and a common cause of PPH [5]. US is the primary modality for the diagnosis of RPOC. On gray-scale US, the presence of a thickened endometrial echo complex (EEC) of at least $10 \mathrm{~mm}$ has a diagnostic sensitivity of $80 \%$ [13] and presence of an endometrial or intrauterine mass has a diagnostic sensitivity of 79\% [14]. Additionally, color Doppler US can further enhance the diagnosis of RPOC as any vascularity detected in a thickened EEC or mass increases the likelihood of RPOC [5].

$\mathrm{CT}$ or magnetic resonance imaging (MRI) can serve as diagnostic adjuncts in complicated cases. However, there is variability of postcontrast enhancement on CT imaging and of T1- and T2-weighted signal intensity depending on the degree of hemorrhage and tissue necrosis $[14,15]$.

Gestational trophoblastic disease is a rare complication of pregnancy encompassing a group of interrelated diseases ranging from premalignant partial and complete hydatidiform mole to malignant diseases of an invasive mole, choriocarcinoma, and rare placental-site trophoblastic tumor and epithelioid trophoblastic tumor [16]. The serum and urine hCG levels are elevated in this disease process. Choriocarcinoma is a rare trophoblastic tumor characterized by myometrial and vascular invasion with high incidence of pulmonary metastasis in the form of nodules with surrounding ground glass opacities [17].

In the present case, the vascularity of the uterine mass in addition to the myometrial invasion suggested a malignant process as opposed to invasive placenta. In addition, the small ground glass pulmonary nodules in the setting of the invasive uterine mass suggested the diagnosis of choriocarcinoma.

Prior reports have described that retained invasive placenta may mimic acquired arteriovenous malformation [18], leiomyomata [19], and endometrial cancer [20]. Therefore, it is important to consider the diagnosis of retained invasive placenta in patients presenting with $\mathrm{PPH}$ who have risk factors for invasive placentation. This is particularly critical prior to performing a D\&C for presumed RPOC, which in cases of retained invasive placenta may result in massive bleeding, necessitating an emergent hysterectomy.

\section{Conflict of Interests}

None of the authors have any relevant conflict of interests to disclose.

\section{References}

[1] T. Angstmann, G. Gard, T. Harrington, E. Ward, A. Thomson, and W. Giles, "Surgical management of placenta accreta: a cohort series and suggested approach," American Journal of Obstetrics and Gynecology, vol. 202, no. 1, pp. 38.e1-38.e9, 2010.

[2] Y. Oyelese and J. C. Smulian, "Placenta previa, placenta accreta, and vasa previa," Obstetrics and Gynecology, vol. 107, no. 4, pp. 927-941, 2006

[3] T. D. Epperly, J. P. Fogarty, and S. G. Hodges, "Efficacy of routine postpartum uterine exploration and manual sponge curettage," The Journal of Family Practice, vol. 28, no. 2, pp. 172-176, 1989.

[4] J. S. Abramowicz and E. Sheiner, "In utero imaging of the placenta: importance for diseases of pregnancy," Placenta, vol. 28, pp. S14-S22, 2007.

[5] M. A. Sellmyer, T. S. Desser, K. E. Maturen, R. B. Jeffrey Jr., and A. Kamaya, "Physiologic, histologic, and imaging features of retained products of conception," Radiographics, vol. 33, no. 3, pp. 781-796, 2013.

[6] L. Sentilhes, F. Goffinet, and G. Kayem, "Management of placenta accreta," Acta Obstetricia et Gynecologica Scandinavica, vol. 92, no. 10, pp. 1125-1134, 2013. 
[7] E. Adler, R. Madankumar, M. Rosner, and S. E. Reznik, "Increased placental trophoblast inclusions in placenta accreta," Placenta, vol. 35, no. 12, pp. 1075-1078, 2014.

[8] R. M. Silver, K. A. Fox, J. R. Barton et al., "Center of excellence for placenta accreta," American Journal of Obstetrics \& Gynecology, vol. 212, no. 5, pp. 561-568, 2015.

[9] M. Tikkanen, J. Paavonen, M. Loukovaara, and V. Stefanovic, "Antenatal diagnosis of placenta accreta leads to reduced blood loss," Acta Obstetricia et Gynecologica Scandinavica, vol. 90, no. 10, pp. 1140-1146, 2011.

[10] G. Calì, L. Giambanco, G. Puccio, and F. Forlani, "Morbidly adherent placenta: evaluation of ultrasound diagnostic criteria and differentiation of placenta accreta from percreta," Ultrasound in Obstetrics and Gynecology, vol. 41, no. 4, pp. 406-412, 2013.

[11] F. D'Antonio, C. Iacovella, and A. Bhide, "Prenatal identification of invasive placentation using ultrasound: systematic review and meta-analysis," Ultrasound in Obstetrics and Gynecology, vol. 42, no. 5, pp. 509-517, 2013.

[12] M. W. F. Rac, J. S. Dashe, C. E. Wells, E. Moschos, D. D. McIntire, and D. M. Twickler, "Ultrasound predictors of placental invasion: the Placenta Accreta Index," American Journal of Obstetrics \& Gynecology, vol. 212, no. 3, pp. 343.e1-343.e7, 2015.

[13] E. Ustunyurt, O. Kaymak, C. Iskender, O. B. Ustunyurt, C. Celik, and N. Danisman, "Role of transvaginal sonography in the diagnosis of retained products of conception," Archives of Gynecology and Obstetrics, vol. 277, no. 2, pp. 151-154, 2008.

[14] K. M. Elsayes, A. T. Trout, A. M. Friedkin et al., "Imaging of the placenta: a multimodality pictorial review," RadioGraphics, vol. 29, no. 5, pp. 1371-1391, 2009.

[15] H. Fleming, A. G. Ostör, H. Pickel, and D. W. Fortune, "Arteriovenous malformations of the uterus," Obstetrics and Gynecology, vol. 73, no. 2, pp. 209-214, 1989.

[16] F. E. M. Froeling and M. J. Seckl, "Gestational trophoblastic tumours: an update for 2014," Current Oncology Reports, vol. 16, article 408, 2014.

[17] S. Martínez-Jiménez, M. L. Rosado-de-Christenson, C. M. Walker et al., "Imaging features of thoracic metastases from gynecologic neoplasms," RadioGraphics, vol. 34, no. 6, pp. 17421754, 2014.

[18] A. Kido, K. Togashi, T. Koyama et al., "Retained products of conception masquerading as acquired arteriovenous malformation," Journal of Computer Assisted Tomography, vol. 27, no. 1, pp. 88-92, 2003.

[19] A. Ekiz, I. Polat, S. Mumusoglu, B. Aydiner, C. Ozdemir, and H. S. Arslan, "A case of placenta increta mimicking submucous leiomyoma," Case Reports in Obstetrics and Gynecology, vol. 2014, Article ID 429406, 3 pages, 2014.

[20] S. Lim, S. Ha, K. Lee, and J. Lee, "Retained placenta accreta after a first-trimester abortion manifesting as an uterine mass," Obstetrics \& Gynecology Science, vol. 56, no. 3, pp. 205-207, 2013. 


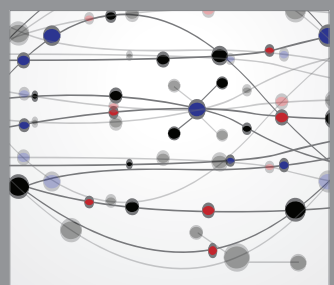

The Scientific World Journal
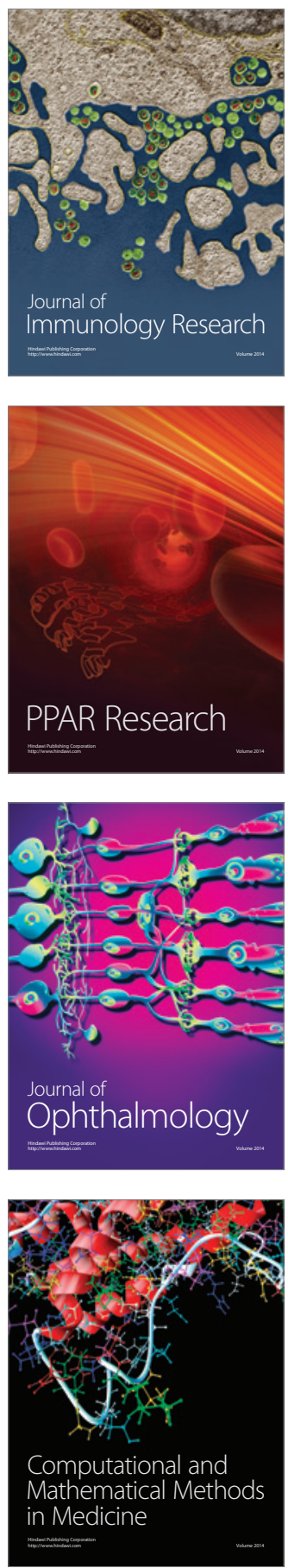

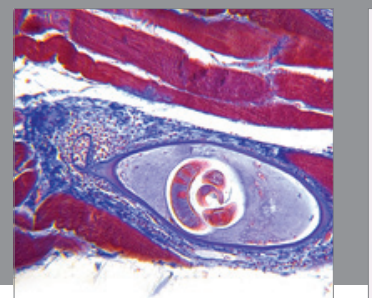

Gastroenterology

Research and Practice
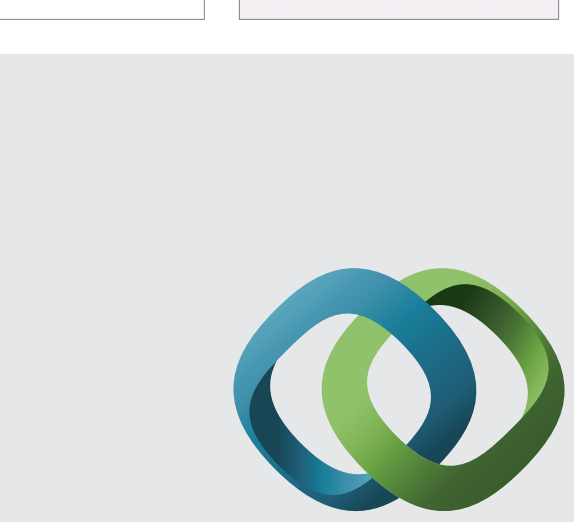

\section{Hindawi}

Submit your manuscripts at

http://www.hindawi.com
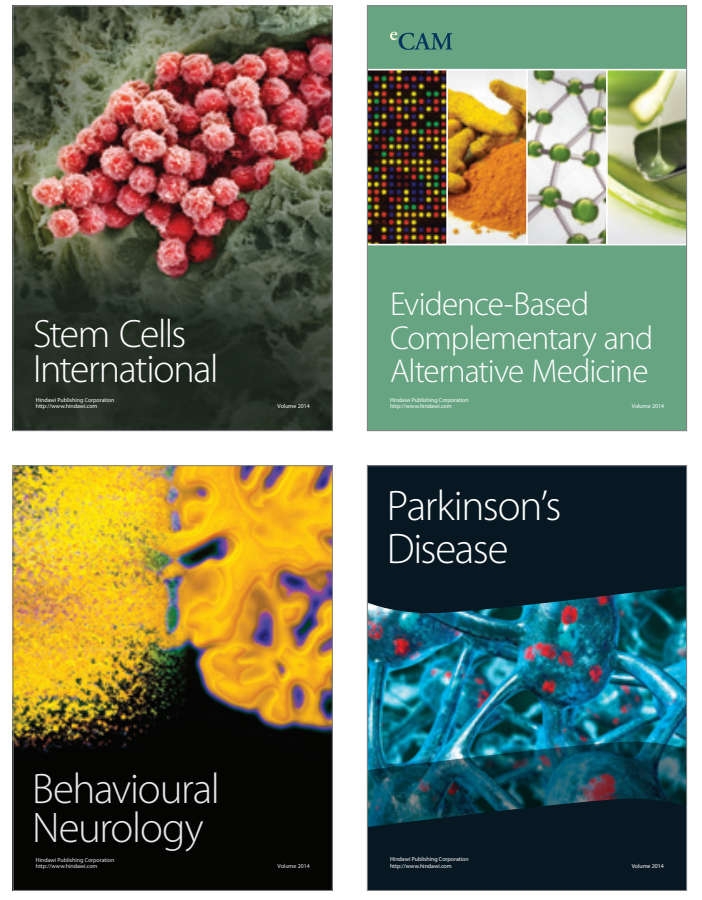
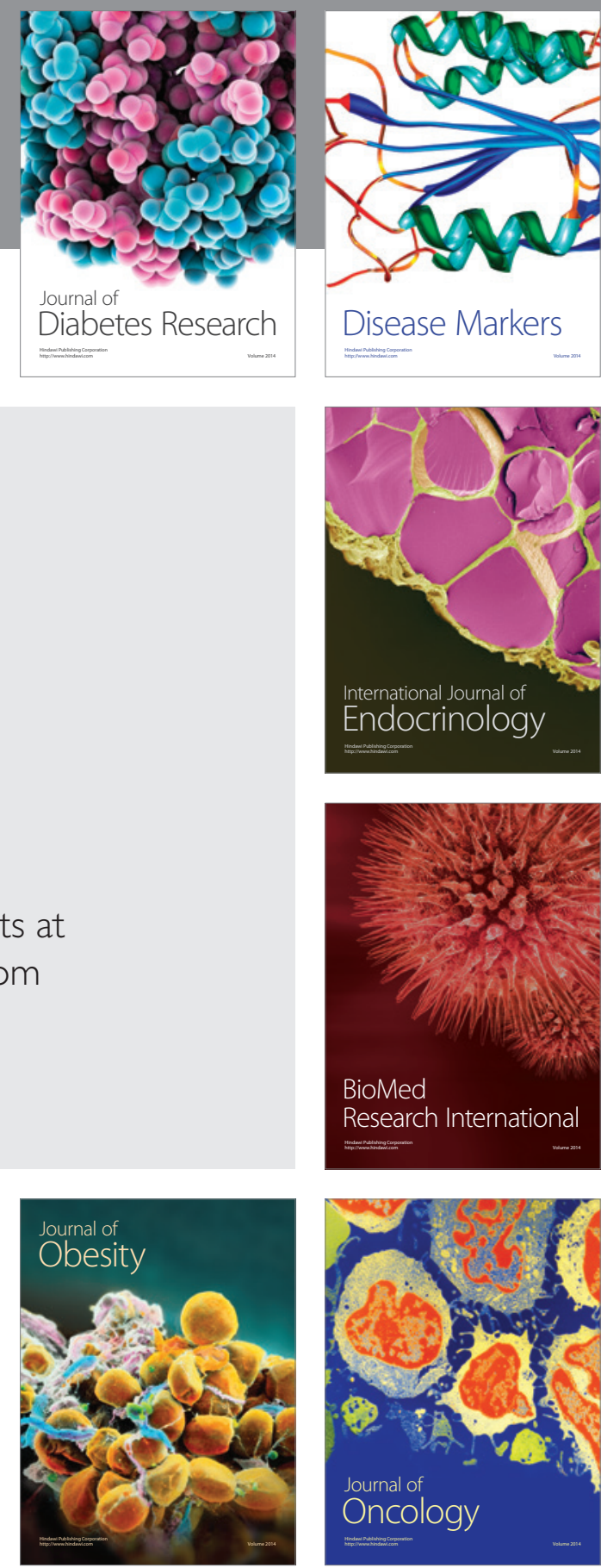

Disease Markers
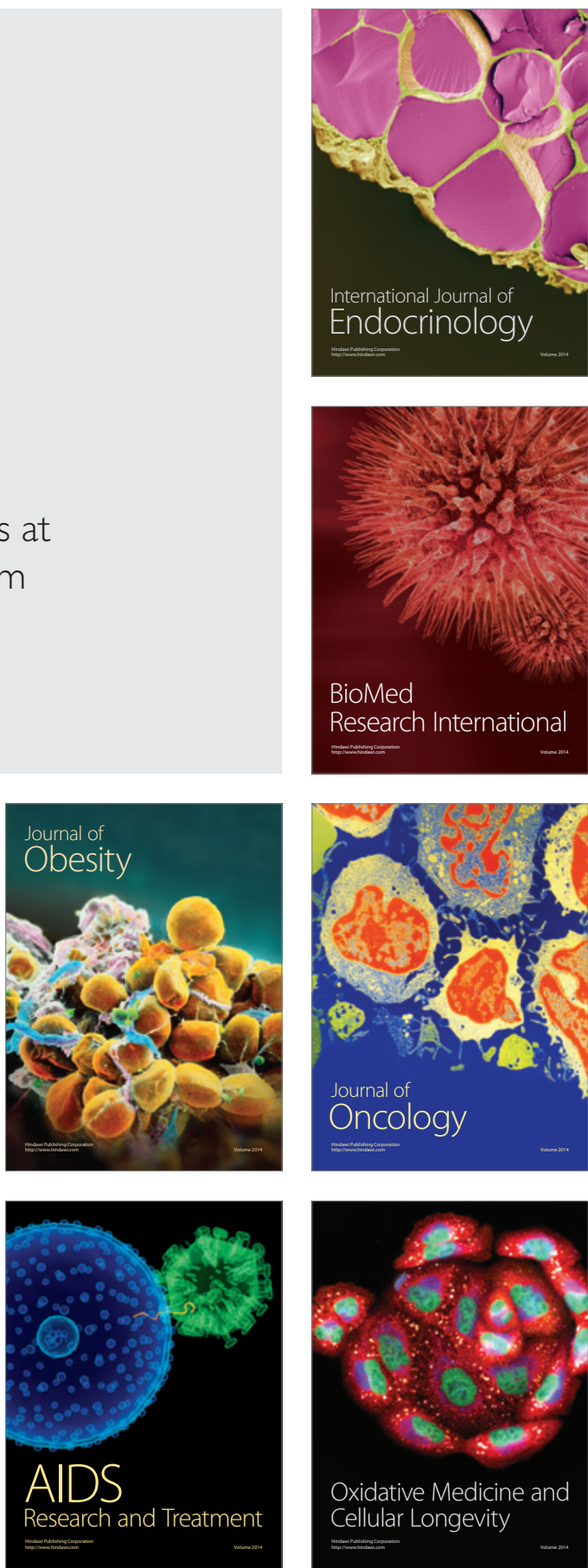